\title{
DIE ROLLE DER BUDDHISTEN IN DER FRÜHINDISCHEN GESELLSCHAFT: ABKEHR VOM KONZEPT DES SOGENANNTEN BUDDHISTISCHEN INDIENS
}

\author{
KATALIN LAKOS \\ Universität Szeged, Lehrstuhl für Alte Geschichte \\ H-6722 Szeged, Egyetem u. 2., Ungarn \\ e-mail: lakoskatalin@gmail.com
}

Der Artikel befasst sich mit der Rolle der Buddhisten in der frühindischen Gesellschaft. Das monolithische Bild eines buddhistischen Zeitalters erfuhr in den letzten Jahrzehnten eine starke Änderung, wobei die sozioökonomischen Prozesse, statt eines großen Sprunges, eher als ein zeitlich und räumlich länger andauernder Entwicklungsprozess beschrieben wurden. Zu dieser Änderung beigetragen haben unter anderem die philologische Forschung, die neuere Datierungsergebnisse der Stadtentwicklung aufgrund der archäologischen Funde in den letzten Jahrzehnten, sowie die ertragreiche und immer noch offene Debatte über die Buddha-Datierung und nicht zuletzt die Ansicht, dass das Zeitalter des historischen Buddha im Vergleich zur traditionellen Auffassung etwas später anzusetzen sei. Das Ziel dieses Artikels ist es, eine neue Lesart des sogenannten buddhistischen Zeitalters wahrnehmbar zu machen.

Schlüsselwörter: Buddhismus, das alte Indien, indische Gesellschaft, buddhistisches Indien, Buddhisten, Wirtschaft und Stadtentwicklung in Indien.

The article deals with the role of Buddhism in the early Indian society. The monolithic image of Buddhist India has been solidly changing during the past decades, and the socioeconomic processes have been described as development processes prolonged through time and space, rather than a big jump. Numerous factors contributed to the formation of this new approach, among others philological research, the latest results based on new archaeological finds concerning the chronology of urban development, as well as the still open debate on the dating of Buddha's life. Last but not least the age of the Historical Buddha, in contrast to the traditional approach, should be dated somewhat later. The aim of this paper is to make discernible a new reading of the so-called Buddhist India.

Key words: Buddhism, early India, early Indian society, Buddhist, urbanisation in early India. 


\section{Einleitung}

Aufgrund der buddhistischen Pāli-Literatur und der traditionellen Buddha-Datierung gingen Rhys Davids und Richard Fick in ihren Werken von einem buddhistischen Zeitalter und einer buddhistischen Gesellschaft aus (Davids 1957; Fick 1897). Dieser Interpretation folgten später mehrere Generationen von Historikern, darunter R. S. Tripathi in seiner mehrere Auflagen erreichten Monographie (Tripathi 1977, S. 102 114) sowie weitere bedeutende Historiker, wie A. L. Basham (1981, S. 46-49), R. S. Sharma (1983, S. 158-168) und Romila Thapar (2002, S. 137-173). Davids und Fick waren ausgezeichnete Philologen, deren Monographien ein zuverlässig erschlossenes und ausgewertetes Quellenmaterial bieten. Dennoch müssen ihre Feststellungen - dank der neueren Forschung, vor allem der modernen Übersetzungen der Texte des PāliKanons, der Pāli- und Prākṛt-Sprachforschungen - an einigen Stellen wohl ergänzt oder geändert werden.

Das monolithische Bild über ein buddhistisches Indien wurde in den späteren wichtigen historischen Werken meist übernommen. In diesen Werken wurde der Zeitraum zwischen den Jahren 600 und 325 vor unserer Zeitrechnung (v. u. Z.) - welcher durch wichtige historisch-politische Ereignisse, wie die Entstehung und Blütezeit des Magadha-Reiches, die Herrschaft der Śiśunāga- und Nanda-Dynastien gekennzeichnet ist - oft als ein von Buddha und den Buddhisten stark geprägtes „buddhistische“ Zeitalter beschrieben. Die klassische marxistische Gesellschaftstheorie „verfeinernd“ haben Kosambi (1969, S. 104-132), Sharma (1983, S. 158-168) und Romila Thapar (2002, S. 137-173) durch die Verwendung und Auswertung komplexen Quellenmaterials - worunter die archäologischen Ergebnisse immer mehr an Gewicht gewonnen haben - ein neues Bild des sogenannten buddhistischen Zeitalters gegeben, welches die Datierungsfragen betreffend flexiblere Interpretationsmöglichkeiten bot, als das in den Werken von Davids und Fick beschriebene Bild. Alle drei haben die Gemeinsamkeit, dass sie dieses Zeitalter im 6.-5. Jahrhundert v. u. Z. verorten und als eine deutlich trennbare Periode in der Geschichte des alten Indiens beschreiben. Die vorliegende Studie hingegen zielt darauf ab ein dynamischeres Bild dieses Zeitalters zu gestalten. Obgleich viele Feststellungen der genannten Autoren bezüglich der markanten technischen, wirtschaftlichen und gesellschaftlichen Veränderungen ihre Geltung haben, können die Problematik der Buddha-Datierung oder die archäologischen Aspekte nicht außer Acht gelassen werden, die die zeitlichen Rahmen des Beginns oder der intensiven Entfaltung dieser Veränderungen modifizieren könnten. Dementsprechend sollte der Fokus eher auf die Rolle des Buddhismus gelegt werden, der sinngemäß einen langwierigen Einfluss auf die frühindische Geschichte ausgeübt hat.

\section{Die Problematik der Buddha-Datierung}

Obwohl die Angaben darüber ungenau sind, galt das Todesdatum des historischen Buddha jahrelang als eine Art Orientierungspunkt der Chronologie der indischen Geschichte. Er soll im Alter von 80 Jahren gestorben sein (Wojtilla 2014, S. 9-23). Eine 
indische Tradition setzt für Buddhas Tod das Jahr 543 v. u. Z. an ${ }^{1}$, aber in der Überlieferung ist eine Lücke von 60 Jahren. Diese 60 Jahre entsprechen genau einem Zyklus in der Zeitrechnung der Inder und anderer asiatischer Völker, der jeweils 60 Jahre umfasste. ${ }^{2}$ Das Jahr 483 v. u. Z. als Todesjahr Buddhas stimmt auch besser mit der Chronologie späterer Ereignisse überein.

Um das Datum des historischen Buddha festzulegen, fand im Jahre 1989 in Hedemünden ein Symposium statt (Bechert 1991/1992/1997). Es ist nicht überraschend, dass in der Datierungsfrage unter den Teilnehmern und weiteren, an den zum Symposium vorliegenden Bänden mitwirkenden Fachkollegen keine Einigung auf eine bestimmte Datierung erzielt werden konnte. Es lassen sich jedoch deutliche Gewichtsverschiebungen gegenüber dem vorausgehenden Stand der Forschung feststellen.

Im Gegensatz zu früheren Theorien verortet die Mehrheit der Forscher - aufgrund der Ergebnisse der neuesten archäologischen und kulturhistorischen Analysen den Todeszeitpunkt von Buddha zwischen den Jahren 420 und 350 v. u. Z. ${ }^{3}$ Eine genaue Zeitdatierung ist auf Grundlage der aktuellen Forschung allerdings nicht möglich. Dementsprechend lassen die Jahre von Buddhas Geburt und Tod auch heute noch eine Reihe von Fragen offen.

Gegen die Glaubwürdigkeit und Akzeptierung der soganennten „kurzen Chronologie“ argumentieren die Forscher, dass diese in der Regel ebenso widersprüchlich sei, wie die sogenannte „korrigierte lange Chronologie“. Weder die „kurze“ noch die „lange Chronologie“ biete zuverlässige und glaubwürdige chronologische Daten. Gebe man allerdings das Datum um 486/480 v. u. Z. als Buddhas Todesjahr auf, so würde die ganze bisherige Chronologie kippen. Es soll hierbei allerdings betont werden, dass die Diskussionen über die Buddha-Datierung keinen Einfluss auf die sozialgeschichtliche Rolle des Buddha und des Buddhismus ausüben, für die Buddha-Biographie sind sie jedoch unerlässlich.

\section{Die wirtschaftliche Lage}

Manche Hypothesen hinsichtlich des Zeitpunktes und der Gründe der Veränderungen in materieller Beziehung und des sozialen Lebens der Bevölkerung von Kosala und Magadha betonen die vorrangige Bedeutung des Gebrauchs von Eisenwerkzeugen für die Urbarmachung und Besiedlung der dicht bewaldeten Gebiete der mittleren Gangesebene seit etwa 600 v. u. Z. (Kosambi 1969, S. 212-213; Sharma 1982, S.

${ }^{1}$ Die Theravāda-Buddhisten in Ceylon (Śrī Lanikā) und Südostasien behaupten, dass der physische Tod des Buddha im Jahr 544/543 v. u. Z. eingetreten ist. Das ist die ursprüngliche, sogenannte ,nicht korrigierte lange“ oder ,südliche buddhistische Chronologie“, die in allen TheravādaGemeinschaften in Süd-und Südostasien heuzutage immer noch verwendet wird.

${ }^{2}$ Siehe darüber Turnour (1837, S. 48-50). Turnour hat in dieser Arbeit die sogenannte ,korrigierte lange Chronologie" gegründet. Diese Berechnung setzt den Tod des Buddha auf das Jahr 480 v. u. Z.

${ }^{3}$ Das ist die sogenannte „,kurze Chronologie“. 
209-222; Thapar 2002, S. 142-144). Einige Forscher schenken dieser Tatsache weniger Bedeutung (Gombrich 1988, S. 49-59).

Die Hypothese, dass eiserne landwirtschaftliche Geräte in Buddhas Zeit in größerer Zahl Verwendung fanden, wird dadurch unterstützt, dass in dieser Zeit begonnen wurde, die Eisenvorkommen in Singhbhum und Mayurbhanj zu erschließen (Sharma 1983, S. 121). Im Zusammenhang mit der Entwicklung der Produktionsverhältnisse in der spätvedischen Zeit betont Mylius die Schlüsselrolle des Eisenerzgewinnes, die Verarbeitung des Eisens und die Rolle der Eisenschmiedetechnik. Allerdings fügt er hinzu, dass die Rolle der Eisenschmiedetechnik in den zeitgenössischen Quellen angeblich nicht dementsprechend betont würde, wie es sich aufgrund der ausgegrabenen Objekte annehmen ließe (Mylius 1971, S. 182-195). Die Eisenverarbeitungstechnologie und die damit verbundene Entwicklung von Schmelzöfen mit höherer Temperatur könnte somit zur Entstehung der Northern Black Polished Ware ${ }^{4}$ (im folgenden: NBPW) geführt haben. Die Bewohner der mittleren Gangesebene hätten demnach den doppelten Vorteil, des reichen Eisenvorkommens einerseits und die Beherrschung der Eisenschmiedetechnik anderseits, erkannt. Dies hat mit großer Wahrscheinlichkeit zu einer vorteilhafteren wirtschaftlichen Lage verholfen, als die der Träger der Painted Grey Ware (im folgenden: PGW) ${ }^{5}$, die Eisen auch bereits verwendet hatten.

Allerdings ist die Anzahl von Verweisen auf eiserne landwirtschaftliche Geräte in den zeitgenössischen Quellen begrenzt. Hierbei ist es eine weitere Schwierigkeit, dass das Alter dieser Referenzen nicht genau zu bestimmen ist, dementsprechend ist eine genaue Datierung der Häufigkeit, der Verbreitung des Eisens und dessen Qualität nicht möglich.

Diese Überlieferungen werfen auch die Frage auf, ob es eine Beziehung zwischen der Entwicklung des technischen und technologischen Know-hows, in unserem Fall der Eisenschmiedetechnik und dem wirtschaftlichen Wohlstand und Überschuss gibt? Einige Forscher sind der Meinung, dass bei den wirtschaftlichen und sozialen Umbrüchen in der Zeit Buddhas Eisen eine unentbehrliche und wesentliche Rolle gespielt habe (Sharma 1982, S. 214-215). Anderseits ist die Urbanisierung auch ohne die Verwendung von Eisen möglich. ${ }^{6}$

Der Hypothese bereitet jedoch die Tatsache Schwierigkeiten, dass die Angaben aus den Texten bezüglich Eisenvorkommen und der Verwendung von Eisengeräten in

${ }^{4}$ Das 5. und 4. Jahrhundert v. u. Z. bezeichnen die Archäologen als die Blütezeit der NBPW, der nördlichen schwarzen polierten Keramik. Dieser Ausdruck kennzeichnet eine Töpferware von vorzüglicher Qualität, die ungefähr seit dem 6. Jahrhundert v. u. Z. (vermutlich als Gefäße für Weine und Öle) für den Handel hergestellt wurde. Man teilt die Zeit der NBPW grob in drei Phasen: die frühe $(550-400$ v. u. Z.), die mittlere $(400-250$ v. u. Z.) und die späte $(250-100$ v. u. Z.) Phase. Die NBPW war in erster Linie eine sogenannte de luxe-Keramik.

${ }^{5}$ PGW ist die Eisenzeit-Kultur der Ganges-Ebene, eine Kultur, die etwa zwischen den Jahren 1200 v. u. Z. bis 600 v. u. Z. existierte. Das ist eine feine graue Keramik mit geometrischen Mustern und schwarzer Lackierung. Die NBPW ist eine Weiterentwicklung der PGW.

${ }^{6}$ Ein gutes Beispiel ist die ägyptische Kultur, wo man die Granitblöcke ohne die Verwendung des Eisens verfertigt und gefestigte Städte gebaut habe. Auch während der Zeit der indischen Harappa-Zivilisation seien herrliche Städte entstanden. 
der landwirtschaflichen Produktion nicht durch archäologische Funde aus der Zeit Buddhas belegt werden können. Manche Forscher behaupten, dass all dies ökologisch zu erklären sei, wonach der feuchte, warme Boden im östlichen Uttar Pradesh und in Bihar zur raschen Korrosion des Eisens führte (Sharma 1982, S. 213-214). Allerdings sollten hierbei einige konkrete archäologische Artefakte, die eindeutig auf das 6.-5. Jahrundert v. u. Z. zu datieren sind, beachtet werden (Gaur 1983, S. 422431). ${ }^{7}$ Und auch die buddhistischen Darstellungen, die dem Beweis für die Beziehung zwischen frühem Buddhismus und dem Pfluganbau dienen können, sollten Erwähnung finden (Wojtilla 1989, S. 94-106). ${ }^{8}$

Es kann also festgehalten werden, dass der wirtschaftliche Überschuss einen zentralen Punkt der ökonomischen Veränderungen bildete. Es ist einerseits einleuchtend, dass die Gründung des buddhistischen Mönchs- und Nonnenordens vom Vorhandensein eines wirtschaftlichen Überschusses, bzw. einer ausreichenden Ernährungsbasis abhängig war. Anderseits den Anreiz, einen wirtschaftlichen Überschuss zu produzieren, dürfte auch die Aussicht auf Handel mit diesem Überschuss geboten haben. Der Handel und die Urbanisierung wären ohne eine starke dörfliche Basis und ausreichenden landwirtschaftlichen Überschuss unvorstellbar gewesen. Immer wenn die hauptsächlichen Beschäftigungen: die Landwirtschaft, der Handel und die Rinderzucht erwähnt werden, nimmt die Landwirtschaft eine Vorrangstellung vor allen anderen ein. Die Vorrangstellung der Landwirtschaft wird aufgrund der Entstehung der neuen sozioökonomischen Gesellschaftsschichten, durch die gahapati(s) und die setṭi(s) vertreten (Shrimali 2014, S. 268).

In der buddhistischen Literatur sind ausgiebige Referenzen über Handelskarawanen, Kaufsmanngilden, Marktstädte und Handelsstraßen zu finden. Aufgrund der Häufigkeit dieser Hinweise kann man vermuten, dass der Handel ein wichtiger Bestandteil des wirtschaftlichen Lebens dieser Zeit gewesen sei. Diese Referenzen bieten nicht nur Einblicke in eine aufstrebende Makroökonomie, sondern berichten auch über die Ausbreitung des buddhistischen Ordens. Der frühe Buddhismus war mit dem Handel und mit der Verbreitung der Handelsbeziehungen stark verbunden.

Archäologische und literarische Zeugnisse untermauern, dass die buddhistischen Texte prosperierende wirtschaftliche Verhältnisse beschreiben, die auf Agrarwirtschaft, Viehzucht, kleine Produktion von materiellen Gütern und beginnende Entwicklung der Geldwirtschaft basieren. Die Produktion diente sowohl dem Lebensunterhalt als auch dem Handel. Es fehlen aber wichtige statistische Daten, die uns ermöglichen ein Bild von der Vernetzung und vom Ausmaß der wirtschaftlichen Verbindungen zu

${ }^{7}$ Unter den gefundenen landwirtschaftlichen Artefakten aus Eisen befinden sich Sicheln, Jätmesser, Pflugscharen und Hacken, die auf die VI. Periode, sprich auf das 6.-5. Jahrhundert v. u. Z. zu datieren sind. Gaur's Meinung zufolge, dürfte Ihre Verwendung die landwirtschaftliche Produktion erhöht haben, um einen wirtschaftlichen Überschuss zu produzieren, der eine wesentliche Rolle bei der Urbanisierung und Urbarmachung gespielt haben könnte.

${ }^{8}$ Das Relief aus Bodh Gaya aus dem 2. Jahrhundert n. u. Z. stellt die Geschichte dar, die auch in der Hatthirājavaṇna Sutta (SamNi I.4.2.) zu lesen ist. In der Erzählung erscheint Māra, der buddhistische Teufel im Körper eines riesigen Elefantenkönigs vor Buddha, der gerade am Ufer des Nerañjanā-Flusses in Uruvelā verweilte. Die Rüssel des Elefantenkönigs sahen aus und waren so groß, wie eine Pflugschar ,nangalaphāla“. 
gewinnen. Der Stil der Beschreibungen in den Texten ist meistens idealisiert. Handwerker wurden in Gemeinschaften aufgeteilt aufgrund des erblichen Charakters ihrer Tätigkeit und es gibt Hinweise auf Gilden (Fick 1897, S. 177-180). All diese Referenzen stammen aus Literaturquellen von mehreren Jahrhunderten, beginnend im 4. Jahrhundert v. u. Z. bis zu den Jātaka-Erzählungen, welche in die ersten Jahrhunderte n. u. Z. reichen. ${ }^{9}$

\section{Die gesellschaftliche Lage: die Soziologie des Buddhismus}

Die Urbanisierung in der Zeit des historischen Buddha führte zu enormen Umbrüchen der bis dahin bestehenden Gesellschaftsordnung und begünstigte auch religiöse Umwälzungen. Die frühere geschlossene Gesellschaft wandelte sich und wurde in gewissem Maße offener. In diesem Zeitraum sind neue Gesellschaftsschichten aufgetaucht, bzw. bekleideten ,alte Schichten“ nun neue gesellschaftliche Rollen.

Die Mehrheit der Forscher ist der Meinung, dass die sogenannte „buddhistische Elite $^{\text {"10 }}$ (Gokhale 1965, S. 392-406) vor allem mit den Mitgliedern der drei bestimmenden Gruppen kommunizierte: den Brahmane(n), prominenten Politikern (auch der König gehörte dazu) ${ }^{11}$ und mit Wohlhabenden (Bailey-Mabbett 2003, S. 48-49).

Das häufige Auftauchen der Begriffe von gahapati(s) ${ }^{12}$ und sețthi(s) ${ }^{13}$ in den buddhistischen Quellen ist ein Beleg dafür, dass der Buddha und der Buddhismus intensive Beziehungen zu den alten, aber mit neuen gesellschaftlichen Rollen bekleideten wohlhabenden Gesellschaftsschichten gehabt haben soll.

Bezüglich der Kaufleute und Händler wird sehr oft behauptet, dass der Buddhismus und andere neue Lehren für die neuen Gesellschaftsgruppen genau die Ethik geboten haben sollen, die sie gerade brauchten (Basham 1988, S. 73). Der Buddhismus unterstützte den Handel. Die Geldwirtschaft, ein wichtiger Teil der kommerziellen Kultur, soll nach mancher Ansicht eine Ergänzung der buddhistischen Werte

\footnotetext{
${ }^{9}$ Nach dem jetzigen Stand unserer Kenntnisse kann man behaupten, dass die chronologische Abgrenzung des bestehenden Pāli-Canons ungefähr das gleiche ist, wie die Texte des Rāmāyaṇa und Mahābhāāata, also zwischen 400 v. u. Z. und 400 n. u. Z. (Shrimali 2014, S. 244).

${ }^{10}$ Der Elite-Status bezieht sich vor allem auf eine wirtschaftliche, politische und religiöse Kategorie.

${ }^{11}$ Ulrich Pagel beschäftigte sich in seinem neuen Vortrag „Buddhist Monks at the Royal Courts of Ancient India“" mit der Rolle der buddhistischen Mönche am königlichen Hof im alten Indien. Seiner Meinung zufolge weisen einige Teile des Mūlasarvāda Vinaya darauf hin, dass in der königlichen Regierung und am königlichen Hof Mitglieder des buddhistischen Sangha in großer Zahl vorhanden waren, auch als königliche Berater (17th Congress of the International Association of Buddhist Studies. Wien, 2014).

${ }^{12}$ Gahapati (skt. grhapati) 'Hausherr', 'Familienoberhaupt'.

${ }^{13}$ Setțhi (skr. śreșthin) 'reicher Kaufmann', 'Gildenherr'. Der gahapati, zusammen mit dem seââhi, war eine soziale und wirtschaftliche Kategorie in der frühen buddhistischen Literatur. Es ist vielleicht nicht ganz verkehrt zu sagen, dass der gahapati eher die Dorf- und Landelite, während der sețthi die städtische Elite bildete. Grundsätzlich repräsentierten sie einen gewissen wirtschaftlichen Status in einer sich transformierenden Agrarwirtschaft auf allen ländlichen Gebieten, die als Versorger der Städte und anderer, in Entwicklung befindlicher Konurbationen erschienen.
} 
gewesen sein, in einer Gesellschaft, wo der Status mehr auf dem Wohlstand und weniger auf der Herkunft beruhte. Die Lehre vom karma rechtfertigte das Vorhandensein von Reichtum und sicherte einen künftigen Verdienst für die gegenwärtige Leistung.

Eine andere Argumentationsweise wäre jedoch ebenfalls möglich, wonach der Buddhismus eine gewisse Abneigung gegen den Anstieg des Handels gezeigt haben soll. Die buddhistische Gemeinschaft habe den Mönchen und ihren Mitgliedern die Verwendung des Geldes und die Akkumulation von Besitz untersagt. In Wahrheit hätten sie also die neuen sozialen Elemente, wie die Liebe zum Geld, das Privateigentum und den Luxus abgelehnt.

Der Buddhismus stellt sich als ein Produkt der Stadtentwicklung dar. Alternative Theorien hingegen verstehen den Buddhismus eher als eine Reaktion gegen die neue städtische Umgebung, wovor es viele in die Wildnis zog um geistige Erlösung zu suchen (Gombrich 1988, S. 58). Anhand von Drekmeiers Konzept über das Trauma des Stammes, liege die Behauptung nahe, dass die Veränderungen in der neuen urbanisierten Gesellschaft schließlich zu einer Art Entfremdung der Menschen führte, und Verlorenheit und Verzweiflung erzeugte. Gombrich verweist hierbei auf die Verbindung zwischen Urbanisation und spiritueller Unpässlichkeit als ein wichtiges Charakteristikum des Buddhismus (Gombrich 1988, S. 57).

\section{Die Stadtentwicklung}

In den buddhistischen literarischen Quellen ist ebenso viel die Rede von Dörfern, wie von großen Städten. Aufgrund der Texte muss davon ausgegangen werden, dass es in der Zeit des historischen Buddha einige große Städte gab ${ }^{14}$, umgegeben von vielen kleinen Dörfern, die sich aus landwirtschaftlicher Produktion ernährten und meistens den Städten dienten. Die buddhistischen Texte kennen eine Hierarchie von Gemeinschaften wie Dörfer, Städte und Länder (Sarao 2010, S. 36-47). Es sind folgende Ausdrücke zu unterscheiden: gāma 'Dorf', nigāma 'Marktflecken, Kleinstadt', janapada 'große Gemeinde, Land', pura 'Stadt', nagara' 'Stadt' und putabhedana ${ }^{17}$ 'eine Stadt mit Marktplatz'. Die Texte lassen jedoch keine präzise Bestimmung der jeweiligen Größe dieser Siedlungstypen zu, nur die Unterscheidung zwischen Städten und kleinen

\footnotetext{
${ }^{14}$ Die sechs größten Städte waren: Sāvatthī, Rājgir, Kapilavatthu, Vesāli, Kosambi und Campā.

${ }^{15}$ Die Begriffe nigāma und gāma werden gelegentlich in den Texten als Synonym verwendet. Die Größe von nigāma liegt jedoch ungefähr zwischen gāma und nagara/rājadhāni. Nigāma bezieht sich aller Wahrscheinlichkeit nach auf eine größere Einheit der Siedlung, als die von gāma (Shrimali 2014, S. 249).

${ }^{16}$ Von den beiden Begriffen, pura und nagara, die urbane Siedlungen bezeichnen, taucht pura seltener in den Pàli-Texten auf (Shrimali 2014, S. 249). Nagaras waren nicht nur im Sinne ihrer hierarchischen Stellung und der Position, die sie besaßen, unterschiedlich, sondern sie haben ein spezifisches Ethos verkörpert (Shrimali 2014, S. 251).

${ }^{17}$ Bezüglich des Begriffes putabhedana siehe Kölver (1985, S. 299-311).
} 
Dörfern. ${ }^{18}$ Das führt zu der Frage, wie die in den Epen und in der buddhistischen Literatur vorhandenen Beschreibungen, als Beweis für die Existenz weit entwickelter und prachtvoller urbaner Zentren in der Lebenszeit Buddhas, mit den archäologischen Beweisen in Einklang zu bringen seien? ${ }^{19}$

Dass es in der Zeit des historischen Buddha Städte gab, wird von den meisten meinungsführenden Forschern akzeptiert. ${ }^{20}$ Zwar waren die meisten Städte von Größe und Bevölkerung her recht klein, spiegeln jedoch anhand der literarischen Texte und archäologischer Funde eine neue Art der Komplexität menschlicher Siedlungen wider: sie waren gleichzeitig sowohl militärische, als auch kulturelle, wirtschaftliche und religiöse Zentren (Sarao 2010, S. 190-191; Erdösy 1985, S. 81-82 und 109-110).

Die Forschung verweist jedoch auch auf die Abwesenheit vieler Funktionen, die normalerweise mit urbaner Zivilisation verbunden werden, und die erst zu einer relativ späteren Zeit zu verorten seien (Sharma 1983, S. 105). ${ }^{21}$ Erdösy identifiziert eine Hochphase der Urbanisierung im Zeitraum von 500 bis 300 v. u. Z. Gekennzeichnet durch blühende Technologie, gebrannte Ziegel, Brunnen, Abwasserleitungen, gepflasterte Straßen und religiöse Denkmäler. Man kann davon ausgehen, dass der Aufstieg des Buddhismus auf den Anfang der zweiten Stufe der Urbanisierung fiel. In den buddhistischen Texten finden sich jedoch keine nachvollziehbaren Hinweise die Stadtplanung oder den Prozess der Urbanisierung betreffend (Erdösy 1988, S. 14). Witzel (2009, S. 287-310) verweist sogar auf das Fehlen von Städten in den frühen buddhistischen Texten. $^{22}$

Es bietet viel Anlass zu Diskussionen, warum die Städte in den vedischen Texten gar keine Erwähnung fanden. Gab es tatsächlich keine oder könnte eher die vermeintliche Abneigung der Brahmanen gegen das Stadtleben sowie die Identifikation des vedischen Lebens mit dem Dorf und seiner Umgebung der Grund dafür gewesen sein, warum Städte in den vedischen Texten weitgehend ignoriert wurden, auch wenn sie existierten. Dürften die vedische Texte von den neuen urbanen Zentren auch deswegen geschwiegen haben, da diese erneute Urbanisation ganz unabhängig von den vedischen Gesellschaften abgelaufen war? Diesbezüglich bietet das Konzept des

${ }^{18}$ Aus den Erzählungen wird von den jeweiligen Orten nichts weiter bekannt, außer ihren Namen. Die Aufenthaltsorte des historischen Buddha geben nur einen gebietlichen Hintergrund für die Predigt und den Verweis auf eine Stadt, aber keine weiteren Informationen.

${ }_{19}$ Prasad gibt in seiner Studie einen Überblick über die theoretischen Paradigmen der Stadtentwicklung in der Zeit des Buddha. Er analysiert die Prozesse aufgrund der literarischen und archäologischen Quellen und betont das Überbewerten der städtischen Bauten aufgrund der buddhistischen Quellen und bezieht sich hierbei vor allem auf den Versuch von M. Lal und G. Erdösy, wonach der Aufbau eines subkontinentalen Modells auf der Grundlage der Feldarbeit in einen oder zwei Bezirken möglich ist (Prasad 2014, S. 214).

${ }^{20}$ Unter anderem Richard Gombrich, Ram Sharan Sharma und Romila Thapar.

${ }^{21}$ Allerdings setzt er auch ein großes Gewicht auf die Urbanisierung im 6. Jahrhundert v. u. Z.

${ }^{22}$ Buddha hätte also den größten Teil seines Lebens in den vorurbanischen Gebieten, Dörfern und Marktplätzen verbracht, und erst in seinem späteren Leben hätte er die sogenannten großen Städte besucht. Nach Witzels Auffassung wurden einige Orte und Städte erst später in den Kanon aufgenommen, manchmal widersprüchlich, manchmal künstlich. Daraus kann geschlussfolgert werden, dass den Beschreibungen der Städte in der frühbuddhistischen Literatur die städtischen Zentren der Maurya-und Post-Maurya Perioden als Beispiel dienten. 
„Greater Magadha“ interessante Anhaltspunkte (Bronkhorst 2007). Bronkhorst behauptet, dass es tatsächlich eine Kultur von „Greater Magadha“ gab, als Gegenkonzept zur Āryavarta ${ }^{23}$, die bis in die Zeit des Grammatikers Patañjali (ca. 150 v. u. Z.) und sogar darüber hinaus von der vedischen Kultur unterschiedlich geblieben war. Der Meinung der Kritiker zufolge versuchte Bronkhorst mit der Idee von „Greater Magadha" eine alternative Geschichtsinterpretation des alten Indiens zu schaffen. Zwar sei das Konzept nicht ganz falsch zu interpretieren, die kulturelle Herkunft von „Greater Magadha“ steht jedoch näher zu den vedischen Hauptrichtungen, als Bronkhorst das vermutet und behauptet (Wynee 2011; Willis 2011, S. 330-332). Nach der Auffassung von Bronkhorst stellt „Greater Magadha“ eine andere historische, soziale und ideologische Entwicklung dar, als die im nordwestlichen Indien. Diese Feststellung ist jedoch nicht ganz neu. Romila Thapar hat schon früher sehr deutlich darauf hingewiesen, dass die Mauryas im Zentrum von Magadha (nicht Greater Magadha) die Jainas, die Ājīvikas und die Buddhisten deshalb unterstützen, da diese heterodoxen Sekten in dieser Region dominant und beliebt waren (Thapar 2000, S. 423). Eine der wesentlichsten Behauptungen Bronkhorsts, wonach die vedische Kultur stark mit den in den Dörfern lebenden Brahmanen verbunden gewesen sein soll, sowie in „Greater Magadha“ in der Entwicklung des Buddhismus die nicht-brahmanischen Stadtbewohner eine Schlüsselrolle gespielt haben sollen, kann aufgrund der buddhistischen Tradition bezüglich der sozialen Zugehörigkeit der städtischen Schüler des Buddha bezweifelt werden. Anhand der Kommentare der Thera und Therī-gāthās stammten mehr als zwei Drittel der Autoren dieser Lieder aus großen Städten und $40 \%$ von denen waren Brahmanen (Gombrich 1988, S. 55-56).

\section{Buddhismus als ein über Jahrhunderte andauerndes Zeitalter}

Das monolithische Bild eines buddhistischen Zeitalters erfuhr eine Änderung, wobei die sozioökonomischen Prozesse, statt eines großen Sprunges, eher als ein zeitlich und räumlich länger andauernder Entwicklungsprozess beschrieben wurden. Zu dieser Änderung beigetragen haben unter anderem die philologische Forschung, darunter die genauere Datierung des Pāli-Kanons und der Kommentarliteratur, die Diskussionen über die Zuverlässigkeit der puranischen Chronologie sowie die neueren Datierungsergebnisse der Stadtentwicklung aufgrund der archäologischen Funde in den letzten Jahrzehnten. Aber auch die ertragreiche und immer noch offene Debatte über die Buddha-Datierung und nicht zuletzt die Ansicht, dass das Zeitalter des historischen Buddha im Vergleich zur traditionellen Auffassung ein wenig später anzusetzen sei.

Diesen oben beschriebenen Entwicklungen in der Forschung sowie der unbefriedigenden Quellenlage ist es geschuldet, dass bei den neuesten Forschungsergebnissen (Olivelle-Leoshko-Ray 2012) ein in gewissem Sinne flexiblerer Umgang mit den Quellen an Akzeptanz gewann, wie es zum Beispiel im Fall von Arthaśāstra zu

${ }^{23}$ Domäne der Arier, wo die vedische Kultur und Religion blühte. 
beobachten ist. ${ }^{24}$ In dieser Hinsicht sollte daher auch bedacht werden, anstatt von den tiefgreifenden wirtschaftlichen und sozialen Auswirkungen und Merkmalen des Buddhismus, eher über ein Zeitalter zu sprechen, das über Jahrhunderte andauerte. Es begann ungefähr um die Geburt des Buddha - muss jedoch nicht fest mit dessen Geburt verbunden sein - und blieb bis zu den ersten Jahrhunderten n. u. Z. stark präsent. Während der Regierungszeit von Aśoka war der Buddhismus keine offizielle Staatsreligion, stand aber auch nicht in politischem Kampf mit dem Hinduismus oder mit anderen Religionen. Er war eine dominante und wichtige Komponente der indischen Gesellschaft. ${ }^{25}$ Nach der Zeit der Mauryas wurde der Buddhismus in Nordwest-Indien sowie in den westlichen und östlichen Teilen des Dekkan schnell zu einer etablierten Religion. In diesen Regionen entwickelten sich die heiligen buddhistischen Zentren entlang der Handelswege sowie in mit Handel stark verbundenen städtischen Siedlungen heraus (Thapar 2000, S. 437).

Zeitliche Obergrenzen sind hierbei wenig nutzbringend. Was mit Sicherheit festgehalten werden kann ist, dass die schriftliche Erfassung des Pāli-Kanons und ihr Gelangen zurück nach Indien, also ca. das 2.-3. Jahrhundert n. u. Z. der Zeitpunkt sein könnte, als die Position des Römischen Reiches und die Weltwirtschaft sich gerade verändert hat, was einen Niedergang der Städte zur Folge hatte.

Die Vermutung, dass der Buddhismus, vor allem in der städtischen Bevölkerung, im Handel und infolge der kulturellen Mission, seine starke Präsenz in der indischen Gesellschaft auch im 2. Jahrhundert n. u. Z. bewahren konnte, erfährt immer mehr Bekräftigung. ${ }^{26}$ Im 3. Jahrhundert v. u. Z., also im Maurya-Reich wurde der Buddhismus ausgehend vom Nordwesten Indiens nicht nur auf dem ganzen indischen Subkontinent sehr schnell bekannt, er bewegte sich auch rasant weiter nordwestlich, Richtung Gandhāra, ins heutige Afganistan und Pakistan. Allerdings gab es auch eine zweite Welle der Verbreitung des Buddhismus im 1.-2. Jahrhundert n. u. Z., dank des Kuschanreiches, das von den nomadischen Yuezhi gegründet wurde. Während der Herrschaft von Kanișka am Ende des 1. und Anfang des 2. Jahrhunderts bewegte sich

${ }^{24}$ Soviel lässt sich sagen, dass sie ihre vorliegende Form nicht früher als im 2. Jahrhundert n. u. Z. gewonnen hat, es ist aber möglich, dass einige Kapitel oder einige Textstellen deutlich älter sind.

${ }^{25}$ Walter Ruben meinte hierzu: „Aśoka empfand sich als Weltverbesserer, als königlicher Missionar, wir würden sagen, als sozialer und politischer Reformer in religiösem Gewand. [...] [Er] wollte [...] das utopische humanistische buddhistische - oder in weiterem Sinne hinduistische - Ideal des guten, gläubigen, mitleidigen Menschen durch moralische Predigt verwirklichen und dabei auch tolerant gegen Brahmanen und ājīvikas sein. Aber er konnte seinen Buddhismus nicht einmal zur Staatsreligion erheben, denn eine solche Einrichtung gab es nicht. Er konnte den Buddhismus auch nicht zur herrschenden Religion machen, keine buddhistische Periode der altindischen Gesellschaftsgeschichte einleiten, er konnte nur scheitern.“ (Ruben 1978, S. 163-164). Auch Thapar meint: „For Aśoka, dhamma was essentially a code of ethical behaviour and the benefits thereof. [...] Therefore, the dhamma of Aśoka was in essence Hindu [...] and the Buddhist ethic was subsumed in it" (Olivelle-Leoshko-Ray 2012, S. 18-33).

${ }^{26}$ Johannes Bronkhorst befasste sich in seinem letzten Vortrag „Why Did the Buddhism and Jainism Develop Differently in India?" mit der Frage, woran die starke Präsenz des Buddhismus in dem Gebiet von „Great Gandhāra“ lag (17th Congress of the International Association of Buddhist Studies, 2014. Wien). 
der Buddhismus von den Gebieten des heutigen Pakistan und Afghanistan weiter östlich und nordöstlich, in Regionen, wo er endlich in der Lage war, große Erfolge zu erzielen: ins Ferghana-Tal oder ins Tarim-Becken. Die prākrit-sprachigen KharoșthīDokumente aus den letzten Jahrzehnten des 3. Jahrhunderts n. u. Z. spiegeln in der Nìya-Oase in Zentralasien, im sogenannten Shanshan Königreich eine Art buddhistische Gemeinschaft wider, in der die wirtschaftliche Rolle der buddhistischen Mönche vorwiegend war (Felföldi 2013). Auf der Insel Sokotra, in der Höhle Hoq gefundene Schriften legen Zeugnis davon ab, dass die Buddhisten bis in das 3.-4. Jahrhundert n. u. Z. am Seehandel aktiv beteiligt waren (Strauch 2012). ${ }^{27}$

Die zeitliche Verortung des indischen Zeitalters vor dem 4. Jahrhundert v. u. Z., die Problematik der Buddha-Datierung inbegriffen, mag schwierig sein. Dennoch sollte der Akzent eher auf die grundsätzlichen Veränderungsprozesse, vor allem die ökonomischen und gesellschaftlichen Entwicklungen gelegt werden, da sie als Wurzel und Triebkraft des Buddhismus die relevanteren Fragen darstellen.

Es kann davon ausgegangen werden, dass der Pāli-Kanon schon vor seiner Niederschrift existierte, wie auch die Jātaka-Erzählungen, die auf alten indischen Märchen basieren, und damit ,zeitlos“ sind. Anders ausgedrückt mag es sein, dass in den früheren Quellen von einem buddhistischen Zeitalter und einem buddhistischen Indien berichtet wurde, der Buddhismus hat aber auch in der Regierungszeit von Aśoka und sogar noch in den späteren Zeiten eine starke Auswirkung gehabt. Daher sollte der Fokus statt auf einem starren Bild eines buddhistischen Indiens, auf die Rolle des Buddhismus in Indien vom 6.-5. Jahrhundert v. u. Z. bis zum 2. Jahrhundert n. u. Z. und sogar darüber hinaus gelegt werden. Der Schwerpunkt sollte also nicht darauf liegen, ob es ein buddhistisches Zeitalter gab - wie es lange fokussiert wurde - sondern darauf, welche Rolle der Buddhismus in der indischen Gesellschaft von der Geburt des Buddha bis ca. 400 n. u. Z. gespielt hat.

\section{Literatur}

Bailey, G. - Mabbett, J. (2003): The Sociology of Early Buddhism. Cambridge.

Basham, A. L. (1981): The Wonder That Was India. Calcutta-Allahabad-Bombay-Delhi. Aufgrund der zweiten verbesserten Ausgabe 1967. Die erste Ausgabe war im Jahre 1954.

Basham, A. L. (1988): The Background to the Rise of Buddhism. In: Narain, A. K. (ed.): Studies in History of Buddhism. Delhi, S. 73-111.

Bechert, H. (1991/1992/1997): The Dating of the Historical Buddha = Die Datierung des historischen Buddha. I. II. III. Göttingen.

Bronkhorst, J. (2007): Greater Magadha: Studies in the Culture of Early India. Leiden (Handbook of Oriental Studies Series 2/19).

Chakravarti, U. (1987): The Social Dimensions of Early Buddhism. Delhi.

Davids, Rhys T. W. (1957): Buddhist India. Calcutta.

${ }^{27}$ Mit Sicherheit kann gesagt werden, dass die Inder spätestens seit dem 1. Jahrhundert v. u. Z. aktiv an Handelsnetzwerken beteiligt waren, die den römischen Westen mit dem Osten, also auch mit Indien verbunden haben. Siehe Székely (2008). 
Erdösy, G. (1985): The Origin of Cities in the Ganges Valley. JESHO Vol. 28, No. 1, pp. 81-110. Erdösy, G. (1988): Urbanisation in Early Historic India. Oxford.

Felföldi, Sz. (2013): Élet a késő ókori, kora középkori Selyemúton. A 3-4. századi Nija a régészeti leletek és az írott források tükrében [Das Leben an der spätantik-frühmittelalterlichen Seidenstraße. Niya in dem 3.-4. Jahrhundert im Spiegel der archäologischen und schriftlichen Quellen]. PhD, Szegedi Tudományegyetem. Szeged.

Fick, R. (1897): Die soziale Gliederung im nordöstlichen Indien zu Buddhas Zeit. Mit besonderer Berücksichtigung der Kastenfrage: vornehmlich auf Grund der Jtaka dargestellt. Kiel.

Gaur, R. C. (1983): Excavations at Atranjīkherā; Early Civilization of the Upper Gangā Basin. Delhi.

Gokhale, B. G. (1965): The Early Buddhist Elite. Journal of Indian History Vol. 42, pp. 392-406.

Gombrich, R. F. (1988): Theravāda Buddhism: A Social History from Ancient Benares to Modern Colombo. London.

Kosambi, D. D. (1969): Das alte Indien: seine Geschichte und seine Kultur. Berlin.

Kölver, B. (1985): Kauțalyas Stadt als Handelszentrum: der Terminus puṭabhedana. ZDMG Vol. 135, pp. 299-311.

Mylius, K. (1971): Die gesellschaftliche Entwicklung Indiens in jungvedischer Zeit nach den Sanskritquellen. Ethnographisch-Archäologische Zeitschrift Vol. 12, pp. 182-195.

Narain, A. K. (2003): The Date of the Historical Sākyamuni Buddha. Delhi.

Olivelle, P. - Leoshko, J. - Ray, H. P. (2012): Reimagining Aśsoka: Memory and History. Oxford.

Prasad, B. N. (2014): Urbanization at Early Vaiśālī. In: Jha, D. N. (ed.): The Complex Heritage of Early India: Essays in Memory of R. S. Sharma. New Delhi, pp. 214-241.

Ruben, W. (1978): Kulturgeschichte Indiens. Ein Versuch der Darstellung ihrer Entwicklung. Berlin.

Sarao, K. T. S. (2010): Urban Centres and Urbanisation: as Reflected in the Päli Vinaya and Sutta Pitakas. New Delhi.

Sharma, R. S. (1980): Ancient India: a Textbook for Class XI. New Delhi, pp. 78-85. Die erste Ausgabe war im Jahre 1977.

Sharma, R. S. (1982): Die Entwicklung der Produktivkräfte und ihre sozialen Folgen in Indien im 1. Jahrtausend v. u. Zeit (unter besonderer Berücksichtigung von Buddhas Zeit). In: Herrmann, Joachim-Sellnow, Irmgard (eds): Sonderausdruck aus Produktivkräfte und Gesellschaftsformationen in vorkapitalistischer Zeit. Berlin, pp. 209-222.

Sharma, R. S. (1983): Perspectives in Social and Economic History of Early India. New Delhi.

Shrimali, K. M. (2014): Literature und Urbanism. In: Jha, D. N. (ed.): The Complex Heritage of Early India: Essays in Memory of R. S. Sharma. New Delhi. pp. 243-279.

Strauch, I. (2012): Foreign Sailors on Socotra: the Inscriptions and Drawings from the Cave Hoq. Bremen.

Székely, Melinda (2008): Kereskedelem Róma és India között [Der Handel zwischen Rom und Indien]. Szeged.

Thapar, Romila (2000): Aśoka and Buddhism as Reflected in the Aśokan Edicts. In: Thapar, Romila (ed.): Cultural Pasts: Essays in Early Indian History. Oxford, pp. 422-438. (Ursprünglich in: Seneviratna, A. (ed.) (1944): King Aśoka and Buddhism. Kandy, pp. 11-26.)

Thapar, Romila (2002): The Penguin History of Early India: from the Origins to AD 1300. London. Tripathi, R. S. (1977): History of Ancient India. Delhi-Varanasi-Patna. (First edition 1942.)

Turnour, G. (1837): The Maháwanso. In Roman Characters. With the Translation Subjoined; and an Introductory Essay on Pāli Buddhistical Literature. In two volumes. Ceylon.

Wagle, N. (1966): Society at the Time of the Buddha. Bombay.

Willis, M. (2011): Review on Johannes Bronkhorst: Buddhism in the Shadow of Brahmanism. Leiden, Brill, 2011 (Handbook of Oriental Studies 2/24). BSOAS Vol. 76, No. 2, pp. 330-332. 
Witzel, M. (2009): Moving Targets? Texts, Language, Archaeology and History in the Late Vedic and Early Buddhist Periods. Indo-Iranian Journal Vol. 52, pp. 287-310.

Wojtilla, Gy. (1989): The Ard-plough in Ancient and Early Medieval India. Remarks on its History Based on Linguistic and Archaeological Evidence. Tools and Tillage Vol. 6, No. 2, pp. 94106.

Wojtilla, Gy. (2014): A Buddha élete és tanítása [Das Leben und die Lehre Buddhas]. In: Szilágyi, Zsolt-Hidas, Gergely (eds): Buddhizmus [Buddhismus]. Budapest, pp. 9-23.

Wynee, A.: (2011): Review on Johannes Bronkhorst: Greater Magadha: Studies in the Culture of Early India. Leiden, Brill, 2007 (Handbook of Oriental Studies Series 2/19). H-Net Reviews in the Humanities \& Social Sciences Online zuletzt abrufbar am 09.11.2014 unter http:// www. h-net.org/reviews/showrev.php?id=31537. 